\title{
DSMC Simulation on Couette Flow in Micro-channels
}

\author{
$\mathrm{Ze} \mathrm{Xi}^{1, \mathrm{a}}$ \\ ${ }^{1}$ School of North China Electric Power University, Baoding 071003, China \\ aCaesarrayxz@gmail.com
}

\begin{abstract}
This paper summarizes the current methods for solving micro-channel flows, and the direct simulation Monte Carlo (DSMC) method is used to simulate the Couette flow, which is the most classical gas micro-flow. The results show that the velocity slip is obvious in the transition regimes and the slip velocity increases along with $K n$ increasing. The simulation results using DSMC are in accordance with the numerical solutions to linearized Boltzmann equation. The DSMC method is effective and easy to carry out in the simulation on gas fluid in micro-channels.
\end{abstract}

Keywords: Couette flow, DSMC method , micro-channel , Boltzmann equation

\section{Introduction}

With the widespread application of micro-electromechanical, micro-channel gas flow has gradually become an important research field. It does not satisfy the common continuum assumption, so it can not be solved by the equation based on the common continuum assumption. The ratio of the mean free path of a gas molecule to the flow feature size is called the Knudsen $(K n)$ number. According to the size of $K n$, flow is divided into four different regions ${ }^{[1]}$.

Euler equation and N-S equation can only describe continuum area, Burnett equation $^{[3]}$ is more suitable than N-S equation, but it can not improve the N-S equation. Numerical methods contain N-S Equation with Velocity Slip and direct simulation Monte Carlo (DSMC) method ${ }^{[4]}$. The high-order slip velocity condition was derived by Besokok ${ }^{[5]}$. In view of this, we use the DSMC method to simulate the micro-channel Couette flow problems. The simulation results are in good agreement with the theoretical results.

\section{The direct simulation Monte Carlo (DSMC) method}

\subsection{Algorithm description}

Direct simulation of Monte Carlo method is to use the appropriate number of analog molecules represent a large number of real gas molecules.

In practice, motion and collision are coupled together, which is difficult to achieve in numerical simulation. If limit DSMC time step less than the average collision time, then in the average sense, a molecule has only one collision in a time step. If command the grid being smaller than the average free path at the same time, the movement and collision of molecules can be dealt with separately. The statistical properties of random state variables such as the state of motion of the simulation molecules in the grid are determined by statistical sampling. The macroscopic flow characteristics are obtained and the simulation of real gas flow is realized. DSMC method does not directly solve the Boltzmann equation, but the simulation of the physical process which were described by the equation. It can 
more easily introduce a more realistic model to achieve the description of complex physical and chemical processes. Simulation of the flow field until the real three-dimensional rare gas flows, which is direct numerical solution Boltzmann equation can not be compared. After years of development, the DSMC method has been widely used in rarefied gas dynamics, and it has been applied in the study of real gas flow and micro-fluidics in the slip flow region and transition region.

\subsection{Calculation step}

First, the simulation molecules are evenly distributed in the grid in the form of random numbers, the initial velocity is given by the Maxwell velocity distribution function:

$$
\left\{\begin{array}{l}
v_{x}=\sqrt{-2 k T \ln (\text { rand }) / m} \cdot \sin (2 \pi \cdot \text { rand }) \\
v_{y}=\sqrt{-2 k T \ln (\text { rand }) / m} \cdot \sin (2 \pi \cdot \text { rand }) \\
v_{z}=\sqrt{-2 k T \ln (\text { rand }) / m}
\end{array}\right.
$$

Where $\mathrm{m}$ is the molecular mass of the gas, $\mathrm{k}$ is the Boltzmann constant, $\mathrm{T}$ is the gas temperature, and rand is the random number entered.

First calculate the new position of each molecule, if the occurrence of diffuse reflection, the formula is amended as:

$$
\left\{\begin{array}{l}
v^{\prime}{ }_{x}=\sqrt{-2 k T \ln (\text { rand }) / m} \cdot \sin x_{1} \cdot \sin x_{2} \\
v^{\prime}{ }_{y}=\sqrt{-2 k T \ln (\text { rand }) / m} \cdot \sin x_{1} \cdot \sin x_{2} \\
v^{\prime}{ }_{z}=\sqrt{-2 k T \ln (\text { rand }) / m} \cdot \cos x_{1}
\end{array}\right.
$$

The model calculates the collision according to the variable hard ball model (VHS) and time-free counter (NTC) method proposed by Bird, and then the flow field is sampled to obtain the macroscopic physical quantity, and the above steps are repeated until the statistical error requirement is satisfied.

\section{Couette Flow}

\subsection{Model establishment}

Couette flow refers to the laminar flow of a viscous fluid between two parallel plates that move relative to each other. The gas flows between a big flat, which has a large ratio of aspect, micro flow flowing large $\mathrm{Kn}$ across the slip flow area to the free molecular region, the wall appeared velocity slip and temperature jump phenomenon. Couette flow is one of the simplest problems in micro-gas flow, which is a valid example to validate the DSMC method.

\subsection{Parameter Settings}

The gas was assumed as Nitrogen. The upper plate's speed was Uw, the lower one was steady. The initial temperature of the two plates and gas were $273 \mathrm{~K}$, the pressure is $101,325 \mathrm{~Pa}$, that was, room temperature and pressure conditions. The total number of simulated gas molecules is 150,000 , with a total of 1,500 meshes and 7500 sub-grids. The grid dimension took $1 / 3$ of the mean free path of the 
molecule. The calculated time step was $1 / 5$ of the average collision time, which was $2.1196 \times 10^{-11} \mathrm{~s}$, which ensured the decoupling of molecular motion and collision. The collision calculation was carried out using Bird's variable hard ball model (VHS) and time-free counter (NTC) method, and the sampling times were 100,000 times.

\section{Results Analysis and Discussion}

\subsection{Velocity}

Figure 1 shows the distribution of the slip velocity, $\mathrm{U}$, as a function of $K n$, calculated by the analytical and DSMC methods, respectively. As can be seen from the figure, the slip velocity increases with the number of $K n$ increases, but the growth rate decreases. When $0.01<K n<0.1$, DSMC method and N-S analytical solution are in good agreement, but when $0.1<K n<0.9$, it shows deviation between the two methods, this is because the analytic method uses N-S equations with slip velocity boundary condition. The results are compared with the numerical solution of the linearized Boltzmann equation in Ref. [1]. The results show that the error of the DSMC is $1.37 \%$ when $0.01<K n<0.1,5.12 \%$ when $0.1<K n<0.9$,method is valid when the error is $1.37 \%$ and the error is $5.12 \%$. The validity of DSMC method is proved.

\subsection{Shea Stress}

Figure 2 shows the DSMC method of flow shear force distribution calculated as the $K n$ number changes from $0.1-0.9$,, it is easy to see that the analytical solution and numerical simulation results are highly consistent, and the slip velocity has the same trend

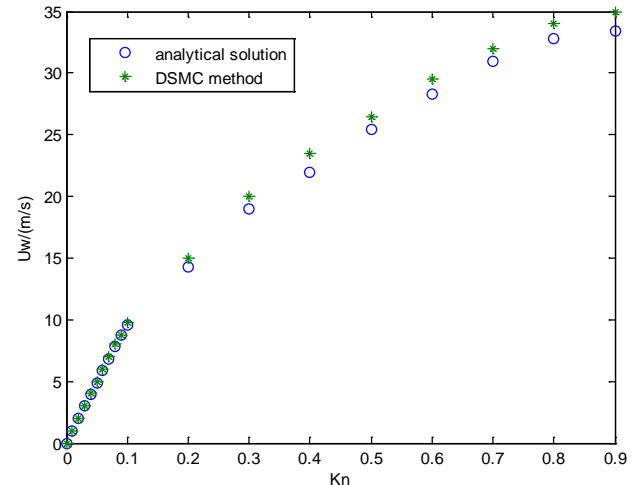

Fig. 1 the distribution of the slip velocity

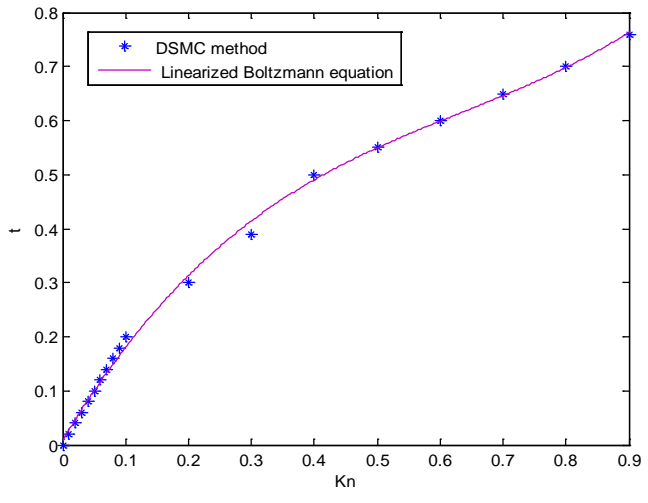

Fig.2 Flow shear force distribution

\subsection{Temperature}

Figure 3 is a schematic view of the profile temperature distribution. In this paper, three $K n$ numbers of 0.05,0.1,0.5 are chosen, and the relative temperature distributions at different cross sections are simulated by DSMC method. It was found that the temperature is quadratic. 


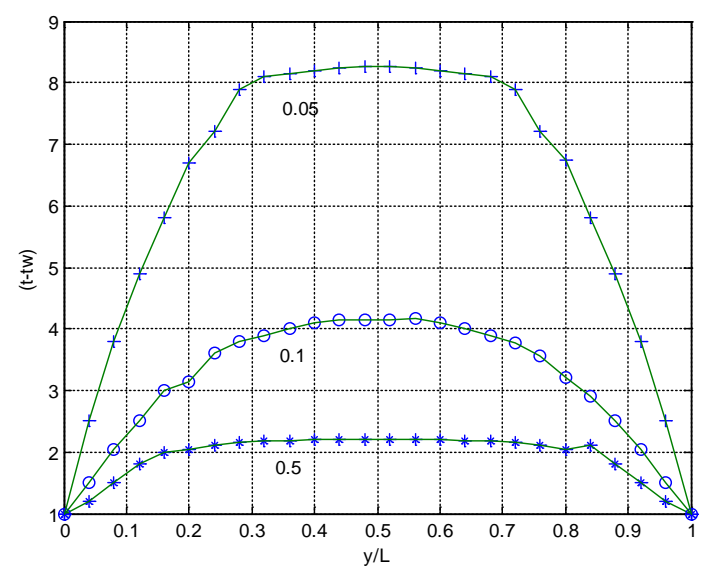

Fig.3 The schematic view of the profile temperature distribution.

\section{Conclusion}

DSMC method in the processing of molecular motion calculation, did not use the deterministic calculation model, but use Monte Carlo calculation method, which greatly simplifies the calculation model; and by using a large number of analog molecules, which ensure the accuracy of the results statistically. The velocity, shear force and temperature distributions of the Couette flow are simulated by DSMS method. The numerical solution of the N-S equation and the linearized Boltzmann equation are compared .

a. The slip velocity and the shear stress change with the $K n$ in the slip flow region and the transition flow region, both of which increase with the increase of the $K n$ and decrease in the growth rate.

b. The distribution of relative temperature at different cross-section was quadratic curve.

\section{References}

[1] Pareschi, Lorenzo. Time Relaxed Monte Carlo Methods for the Boltzmann Equation[J].SIAM Journal on Scientific Computing, 2001, 23(4):1253-1273.

[2] Abdou M A. Chapman-Enskog-maximum Entropy Method on Time dependent Neutron Transport Equation[J]. Journal of Quantitative Spectroscopy and Radiative Transfer, 2006, 101(2):210-225.

[3] Jin S, Slemrod M. Regularization of the Burnett Equations for Rapidgranular Flows via Relaxation[J]. Physica D, 2001,150:207-218.

[4] Bird G A. Molecular Gas Dynamics and the Direct Simulation of Gas Flows [M]. Oxford: Clarendon Press, 1994.

[5] Beskok A. Simulation and Models for Gas Flows in Microgeometries[D]. New Jersey: Princeton University,2009.

[6] SHEN Ching. Information Preservation(IP) Method Insimulation of Internal Rarefied Gas Flows in Mens [J]. 2006,36(1):142-150.

[7] Azad Qazi Zade. A detailed comparison between Navier-Stokes and DSMC simulationsof multicomponent gaseous flow in microchannels[D]. New Jersey: Princeton University,2015. 